\title{
SENTINEL-2 IMAGERY FOR MAPPING AND MONITORING IMPERVIOUSNESS IN URBAN AREAS
}

\author{
G. Kuc ${ }^{1, *}$, J. Chormański ${ }^{1}$ \\ ${ }^{1}$ Warsaw University of Life Sciences, Faculty of Civil and Environmental Engineering, Warsaw, Poland - g.kuc@levis.sggw.pl
}

Commission I, WG I/10

KEY WORDS: sentinel-2, planet scope, imperviousness, climate change, urban areas

\begin{abstract}
:
Nowadays there is need to take the action to find out the innovative solutions to reduce the negative effects of climate change in urban areas. Cities face multiple challenges in water management and flood protection at local scale, especially given an uncertain future climate and a rapidly growing population. The density of impervious surfaces (roofs and pavement) is a key element in evaluating hydrologic processes and occurring the surface runoff - the major component of water cycle in urban catchment. Satellite imagery are powerful and up-to-date data for estimation human impact on urban space. It is also highlighted the possibility of using those results in urban development studies according to adaptation cities to climate change. The Copernicus SENTINEL constellation brings out the potential to provide high quality and free of charge satellites images that enable to estimate parameters related to urban structure and imperviousness. Multitemporal Sentinel-2 data are widely used to calculate the indices for urban sprawl. The normalized difference built-up index (NDBI) and normalized difference vegetation index (NDVI), indicators providing information on urban heat Island effect, are useful for change detection of imperviousness percentage in urban areas. The main objective of this research is fusion of multi-temporal and multi-resolution SENTINEL-2 MSI data for developing a new, innovative approach for improving water management in urban catchments at local scale provide valuable input data for hydrological model. The results obtained were compared by quantitative comparison with Imperviousness HRL of Copernicus Program, visual comparison with OpenStreetMap data as well as qualitative comparison with the high resolution Planet's.
\end{abstract}

\section{INTRODUCTION}

Due to the climate change, it is necessary to take measures to mitigate the negative effects. The most severe changes occur in rapidly developing urban centers. The density of impervious surface plays a key role in shaping hydrologic processes and affects on water cycle in urban areas. (Shuster et al., 2015) Howevere, for analysis purposes, there is need to use the open data and open-source software for satellite images. The Copernicus SENTINEL constellation and other satellite based products are high quality free of charge data capable of estimating parameters related to urban areas and environmental issues (Cornelia, 2018). Additionally, the ESA Sentinel Toolbox applications (SNAP) is a perfect tool for Earth Observation processing and analysis due to its innovative functionality and free downloading. (ESA, 2019) The SENTINEL satellites also provide the ability of independent validation of the presented results because the access to the database is the same for everyone. (Christiansen et al., 2018) Currently, some operational products, such as the "Copernicus High Resolution Imperviousness Layer", are available free of charge to assess required information, but the frequency of their updates is still very limited (every 3 years). High resolution satellite images makes available data with short time revisits and find practical application in almost every scientific field (Lefebvre et al., 2016). The most common and widely used index for monitoring land use change is The Normalized Difference Vegetation Index (NDVI). Calculating satellite derived NDVI is often difficult, however, as SENTINEL-2 imagery is inherently noisy due to cloud and atmospheric contamination, data processing failures, and instrument malfunction. (Robinson et al., 2017) Another important indicator in urban area mapping is the Normalized Difference Build-up Index which could be calculated using SENTINEL-2 imagery. This indicator is also sensitive to cloudiness but research indicates that it can be used as a complementary indice of surface urban heat island effects to the traditionally applied NDVI. (Macarof and Statescu, 2017) The other advantage is 12day repeat cycle so data can be used for solving various environmental problems. The biggest challenge is to combine these resources in such a way that it be possible to get the most reliable information about impervious surfaces in urban spaces. Another task is to find a valuable source of information for validation research results. That could be achieved by comparison with high-resolution data (Planet Scope) and other Copernicus products such as High Resolution Imperviousness Layer.

\section{STUDY AREA}

The study area is located in Warsaw (Fig. 1) - the largest city and capital of Poland. The metropolis stands on the Vistula River and its population is estimated at 1.78 million residents within a greater metropolitan area of 3.1 million residents, which makes Warsaw the 8-th most populous capital city in the European Union.

\section{DATA}

This study used a Sentinel-2 scene (date of acquisition: 08.08.2018), Planet Scope imagery and the Copernicus HRL imperviousness for the year 2015. Additionally, the Google Earth HR imagery and Open Street Map data were used for visual interpretation of the results. All image processing and analyses was carried out in ESA SNAP Desktop and QGIS Desktop. 


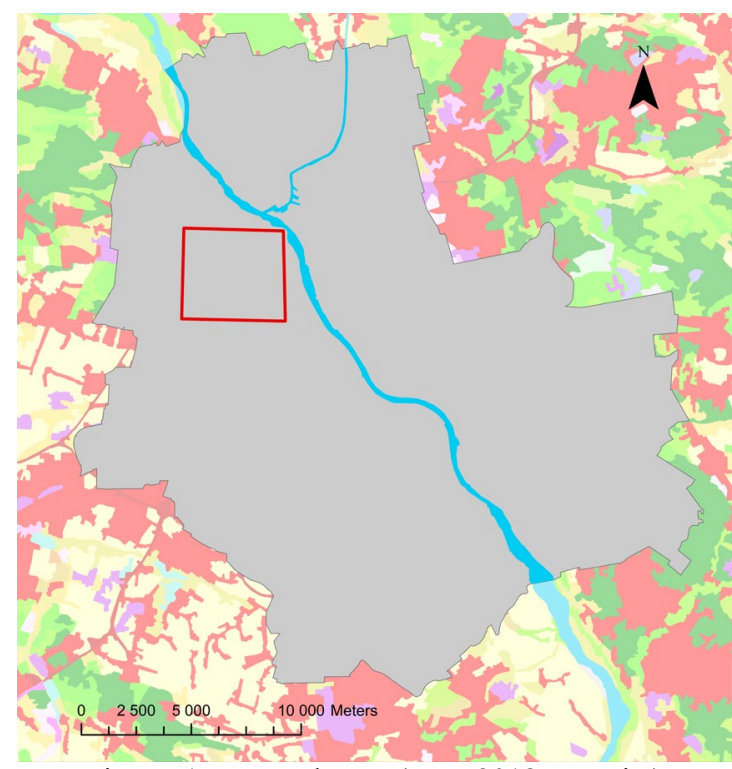

Figure. 1. Research area (CLC 2018, GUGiK)

\subsection{Sentinel-2 imagery}

SENTINEL-2 is a European wide-swath, high-resolution, multispectral imaging mission. The full mission specification of the twin satellites flying in the same orbit but phased at $180^{\circ}$, is designed to give a high revisit frequency of 5 days at the Equator. SENTINEL-2 carries an optical instrument payload that samples 13 spectral bands: four bands at $10 \mathrm{~m}$, six bands at $20 \mathrm{~m}$ and three bands at $60 \mathrm{~m}$ spatial resolution. The orbital swath width is $290 \mathrm{~km}$. The main objectives of this mission are systematic global acquisitions of high-resolution, multispectral images allied to a high revisit frequency, continuity of multispectral imagery provided by the SPOT series of satellites and the USGS LANDSAT Thematic Mapper instrument and observation data for the next generation of operational products, such as land-cover maps, land-change detection maps and geophysical variables. These high-level objectives, determined after consultation with users, will ensure that SENTINEL-2 makes a significant contribution to Copernicus themes such as climate change, land monitoring, emergency management, and security. (ESA)

\subsection{Copernicus High Resolution Layers - Imperviousness Degree}

Pan-European High Resolution Layers (HRL) provide information on specific land cover characteristics, and are complementary to land cover / land use mapping such as in the CORINE land cover (CLC) datasets (Fig. 2). The HRLs are produced from satellite imagery through a combination of automatic processing and interactive rule based classification. Since the production of the 2015 reference year the production is increasingly based on time series of satellite images from a number of different sensors, including the combination of optical and radar data. The main source is the Sentinel Satellites (in particular Sentinel-2 and Sentinel-1). In addition to high resolution (HR) data, since 2015, very high resolution (VHR) imagery was used for some of the products. (ESA, 2019)

The imperviousness products provide information on the percentage and change of soil sealing. Built-up areas are characterized by the substitution of the original (semi-) natural land cover or water surface with an artificial, often impervious cover. These artificial surfaces are usually maintained over long periods of time. The imperviousness HRL captures the spatial distribution of artificially sealed areas, including the level of sealing of the soil per area unit. The level of sealed soil (imperviousness degree $1-100 \%$ ) is produced using a semiautomated classification, based on calibrated NDVI. (ESA, 2019)

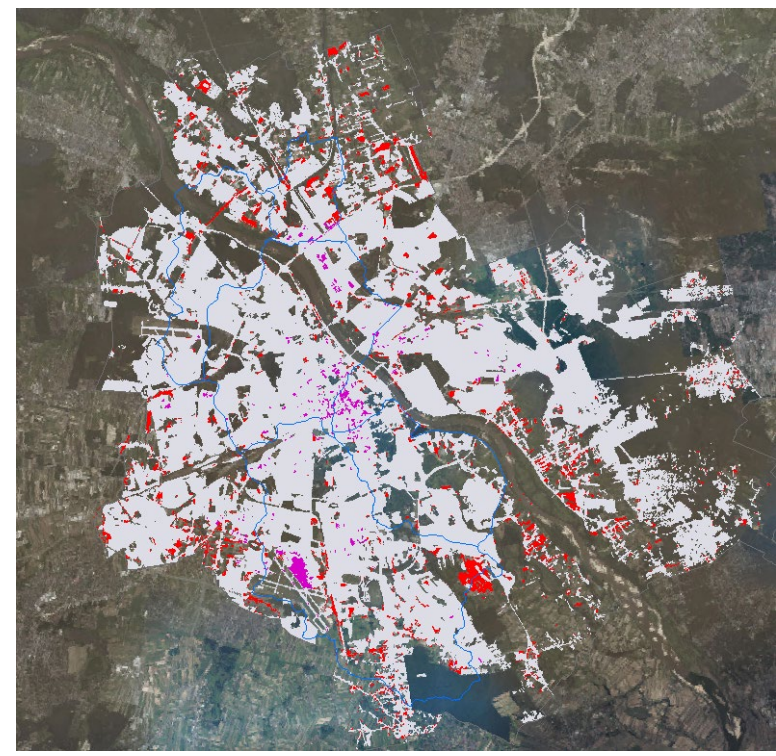

Figure 2. Imperviousness Layer for Warsaw. (Copernicus Program, 2015)

Imperviousness data is available for the reference years 2006, 2009, 2012 and 2015, and contains two types of products: status layers $(20 \mathrm{~m}$ and $100 \mathrm{~m}$ spatial resolution) and change layers for each of the 3-year periods between the 4 reference years (2006$2009,2009-2012,2012-2015$ and in addition for the period 2006-2012). (ESA, 2019)

\subsection{Planet Scope imagery}

Planet Scope satellite imagery is captured as a continuous strip of single frame images known as "scenes". Scenes may be acquired as a single RGB (red, green, blue) frame or a splitframe with a RGB half and a NIR (near-infrared) half depending on the capability of the satellite. In this research, Planet Scope Analytic Ortho Scene was used in order to urban areas mapping. This product is orthorectified, multispectral data from the satellite constellation that have been processed to allow researchers to provide information products for data science and other purposes.

Planet Scope imagery are designed for a wide variety of applications that require imagery with an accurate geolocation and cartographic projection. The imagery has been processed to remove distortions caused by terrain and can be used for many data science and analytic applications. It eliminates the perspective effect on the ground (not on buildings), restoring the geometry of a vertical shot. The Planet Scope Analytic Ortho Scene is optimal for value-added image processing such as land cover classifications. The imagery has radiometric corrections applied to correct for any sensor artifacts and transformation to at-sensor radiance. (Planet, 2019) 


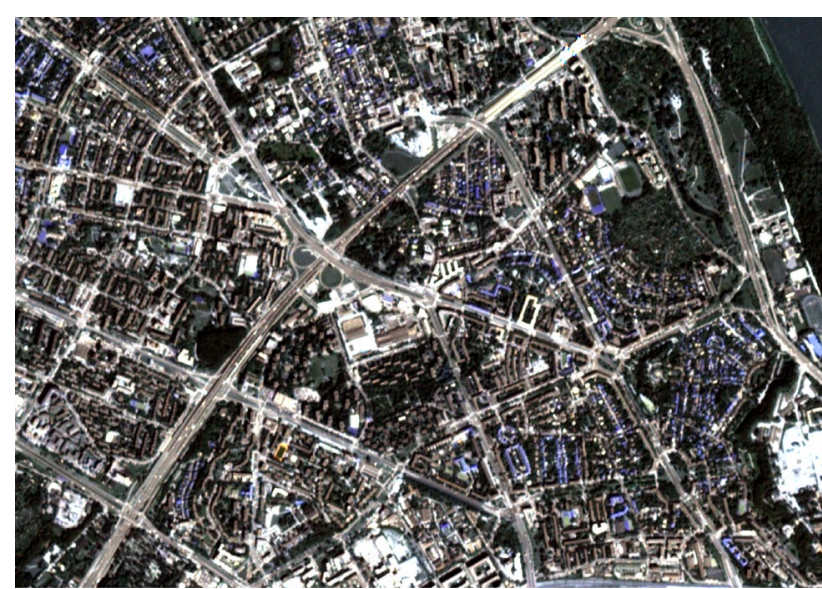

Fig. 3. Example of high-resolution Planet Scope image - the part of Warsaw city (Planet, 2018)

\section{METHODOLOGY}

\subsection{Build-up areas mapping with NDVI}

The NDVI ratio can be determined from the contribution of visible wavelength and near-infra-red wavelengths. The first step included cloud and cloud shadows detection. This step is very important because clouds may impact on NDVI results. For this purpose, the cloud mask prepared by ESA was used to remove clouds from imagery.

Afterwards, the NDVI was computed with the well-known method as follows:

$$
N D V I=\frac{N I R(B 8)-\operatorname{Red}(B 4)}{N I R(B 8)+\operatorname{Red}(B 4)}
$$

Then, impervious mask was obtain using threshold values for NDVI.

\subsection{Build-up areas mapping with NDBI}

The Normalized Difference Build-up Index (NDBI) highlights urban areas with higher reflectance in the shortwave-infrared spectral range (SWIR).

Therefore, the NDBI was computed as follows:s:

$$
N D B I=\frac{S W I R(B 11)-N I R(B 8)}{S W I R(B 11)+N I R(B 8)}
$$

In case of SENTINEL-2 imagery, the SWIR band is $20 \mathrm{~m}$ resolution, so before the calculation, it was need to resample data to lower resolution. Therefore, the final result will be also obtained with a resolution of $20 \mathrm{~m}$.

\subsection{Urban areas identification with Planet Scope imagery}

Planet Scope Analytic Ortho Scene allows to produce 4-band multispectral images (blue, green, red, near-infrared). Due to the limited range of bands, it was decided to use the one of the most known and uses indicators for mapping urban areas Normalized Difference Vegetation Index.

The NDVI is calculated in similar way as for SENTINEL-2:

$$
N D V I=\frac{N I R-R e d}{N I R+R e d}
$$

\section{RESULTS}

This study is concentrated on mapping urban areas so all the mapped land covers were classified into only two categories: impervious surface and all others. At the beginning, the accuracy of the impervious surface classification was assessed mainly spatially. The spatial discrepancy between the mapped and actual boundaries on Open Street Map Layers was illustrated in figure 4 and figure 5 .

It shows that the vectorised boundary of the mapped built-up areas matches closely with the actual border of built-up areas in this part of the image.

The results obtained shows that all of the calculated indices/ data produced could be suitable for urban areas detection. An initial analysis and a visual assessment based on Open Street Map Data indicates that both NDVI (Fig. 4) and NDBI (Fig. 5) indices are a reliable source of information about surface impervious in urban spaces.

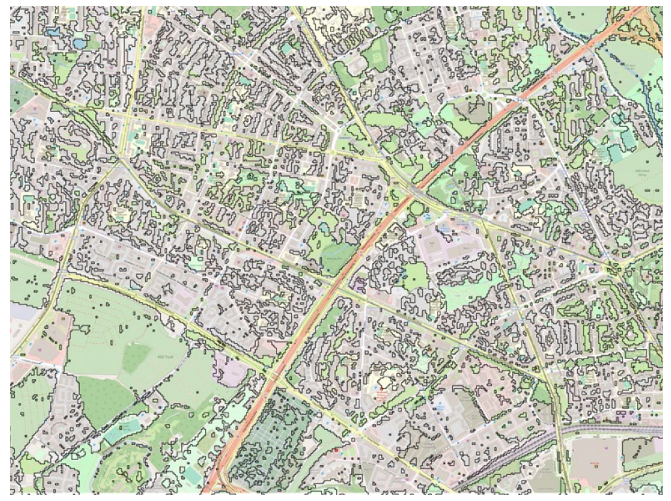

Fig. 4. Comparison of mapped impervious surface based on NDVI with Open street map.

The results also indicate that boundary of impervious surface calculated in basis of NDVI matches more closely with mapped imperviousness in Open Street Map than the same analysis based on NDBI. It could be caused by lower resolution of B11 band $(20 \mathrm{~m})$ compared to other Sentinel-2 bands (10 $\mathrm{m})$.

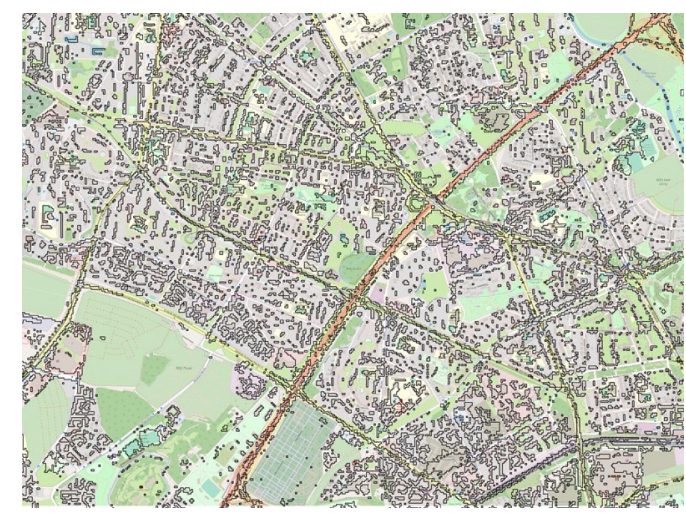

Fig. 5. Comparison of mapped impervious surface based on NDBI with Open street map.

In the next step, the results obtained were compared with highresolution Planet Scope imagery. The spatial discrepancy 
between these collections was illustrated in figure 6 and figure 7 .

As we can observed, the medium value for NDVI was appropriated for urban areas and bare soil in comparison with high-resolution satellite imagery Planet Scope. That was the best result for classification urban areas with using SENTINEL imagery.

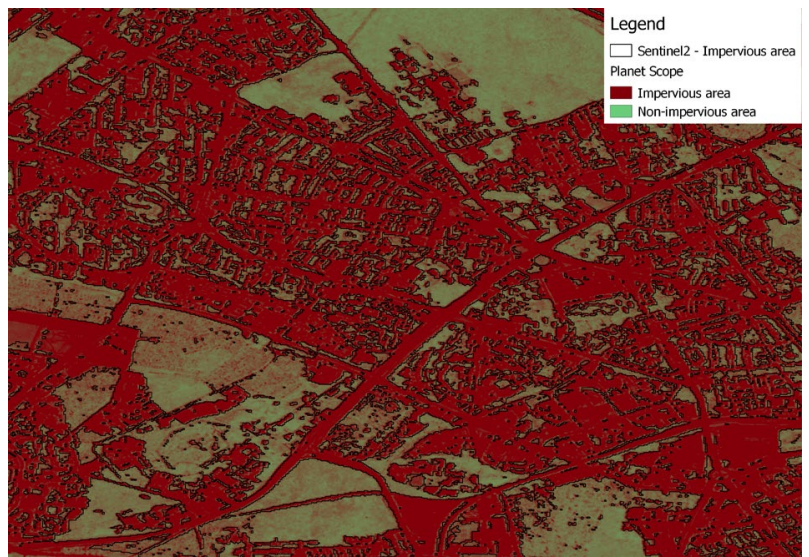

Fig. 6. Comparison of SENTINEL-2 NDVI products with Planet Scope.

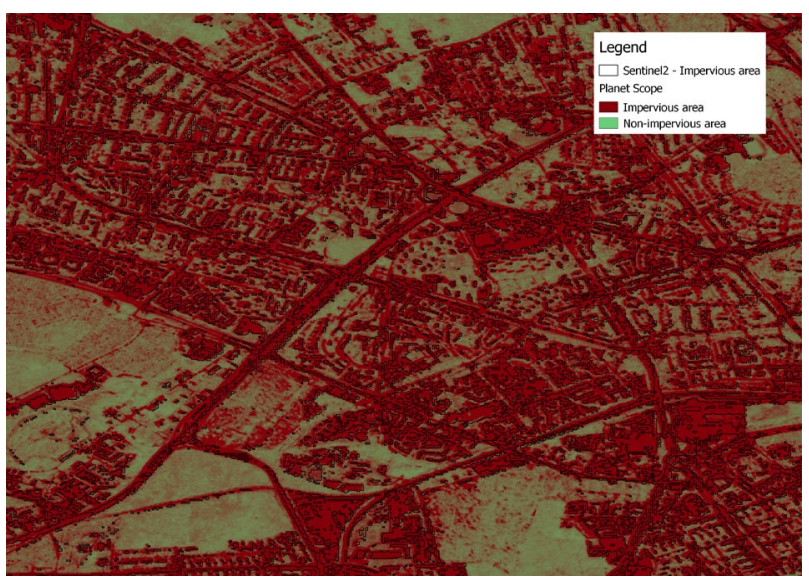

Fig. 7. Comparison of SENTINEL-2 NDVI products with Planet Scope.

Based on the map above, some errors can be seen in the classification of impervious areas. It mainly concerns the areas with lower building density with a greater predominance of green areas. However, areas with high building density and impervious surfaces were classified appropriated.

Then, the best results of mapping impervious surfaces based on NDVI were compared with the Copernicus High Resolution Layers (Fig. 8). There was identified more significant difference between two resources. HRL data are less detailed in comparison to NDVI computed in basis of SENTINEL-2 imagery.

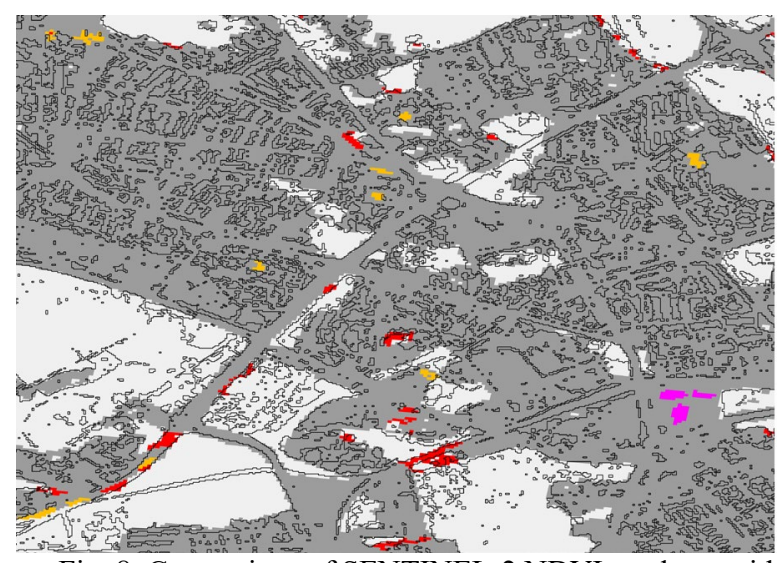

Fig. 8. Comparison of SENTINEL-2 NDVI products with Copernicus HRL

As can be seen on the map above, some of the new impervious areas that were not included in the HRL data were also identified based on SENTINEL-2 imagery.

\section{DISCUSSION}

The objective of this research was to enhance the differentiation between built-up and non-built-up areas by processing SENTINEL-2 data. The result obtained shows that the better accuracy was acquired for the NDVI based urban mapping. The lower spatial compatibility for NDBI results may be independent on resolution of bands using for calculating this indicator. A quantitative comparison of the results obtained with the Imperviousness Degree provided in frame of Copernicus Program may be difficult due to the time discrepancy. The HRL data are available for the year 2015, while the satellite images were acquisited for the year 2018. However, visual comparative analysis indicates that impervious surfaces identified on the basis of satellite images are more detailed than Copernicus products.

\section{CONCLUSION AND FUTURE WORK}

This study could demonstrate the value of SENTINEL data for identification of build-up areas. The results revealed that calculated indicators and mapping urban footprints with SENTINEL-1 imagery are suitable for analysis the imperviousness surfaces in rapidly developing cities. Using this data we can obtain similar results to high-resolution imagery that are very cost-consuming. The best result was acquired for NDVI indicator. In case of NDBI it is need to improve proposed methodology to succeeding obtain better results. Future work will concern further refinement of the methodology for identification and classification of impermeable surfaces using satellite data SENTINEL and other methods. It also includes the comparison of the results obtained with high-resolution UAV data.

\section{REFERENCES}

Cornelia, Ana Maria, 2018. Advantages of Identifying Urban Footprint using Sentinel-1. FIG Congress 2018 Embracing our smart world where the continents connect: enhancing the geospatial maturity of societies. Istanbul, Turkey, May 6-11, 2018.

Lefebvre, Antoine \& Sannier, Christophe \& Corpetti, Thomas, 2016. Monitoring Urban Areas with Sentinel-2A Data: 
Application to the Update of the Copernicus High Resolution Layer Imperviousness Degree. Remote Sensing. 8. 606. $10.3390 /$ rs8070606.

Macarof P., Statescu F., 2017. Comparasion of NDBI and NDVI as Indicators of Surface Urban Heat Island Effect in Landsat 8 Imagery: A Case Study of Iasi. Present Environment and Sustainable Development. DOI: https://doi.org/10.1515/pesd-2017-0032

Planet Team (2017). Planet Application Program Interface: In Space for Life on Earth. San Francisco, CA. https://api.planet.com

QGIS Development Team (YEAR). QGIS Geographic Information System. Open Source Geospatial Foundation Project. http://qgis.osgeo.org

Robinson, N.P.; Allred, B.W.; Jones, M.O.; Moreno, A.; Kimball, J.S.; Naugle, D.E.; Erickson, T.A.; Richardson, A.D. A Dynamic Landsat Derived Normalized Difference Vegetation Index (NDVI) Product for the Conterminous United States. Remote Sens. 2017, 9 863. DOI: doi.org/10.3390/rs9080863

Shuster, William \& Bonta, J \& Thurston, H \& Warnemuende, E \& R. Smith, D. (2005). Impacts of Impervious Surface on Watershed Hydrology: A Review. Urban Water Journal URBAN WATER J. 2. 263-275. 10.1080/15730620500386529.

SNAP - ESA Sentinel Application Platform v2.0.2, http://step.esa.int 